\title{
Electron Energy-Loss Spectroscopy with a Monochromated TEM
}

\author{
W. Grogger, G. Kothleitner, B. Schaffer, F. Hofer
}

Research Institute for Electron Microscopy, Graz University of Technology, A-8010 Graz, Austria

The characterization of nanostructured devices and functional materials at a nanometer scale is paramount for the understanding of the physical or chemical properties. With the introduction of the monochromated transmission electron microscope equipped with a high resolution energy filter it becomes possible to solve advanced materials science problems which have not been accessible before. In particular, one can obtain improved information not only about the chemical composition of a sample, but also about chemical bonding and optical properties.

For the experiments presented here an FEI Tecnai F20 microscope was used $(200 \mathrm{kV}$, Schottky emitter). The system is equipped with a pre-specimen monochromator (Wien filter) [1], a high resolution imaging filter (Gatan) [2] and a better stabilized high voltage supply.

With this setup it is possible to record EELS-spectra with an energy-resolution in the 100-200 meV range (Fig.1) [3, 4]. The improved energy resolution opens new possibilities for studying detailed electronic structure and bonding effects evaluated from near edge fine structures $[5,6]$, band gap [7] and dielectric function measurements via the low-loss part of the spectrum. A typical example for demonstrating the potential of high resolution EELS in materials science is shown in fig.2. The first peak of the $\mathrm{Al} \mathrm{L}_{2,3}$ edge of $\alpha-\mathrm{Al}_{2} \mathrm{O}_{3}$ shows a separation of $0.49 \mathrm{eV}$ which can be attributed to spin-orbit splitting $\left(\mathrm{L}_{3}\right.$ and $\left.\mathrm{L}_{2}\right)$ [8]. These results agree with $\mathrm{x}$-ray absorption spectroscopy measurements. With STEM imaging we could acquire EELS spectra with an energy resolution of less than $0.25 \mathrm{eV}$, but the spatial resolution was limited by the properties of the monochromated illumination system of the TEM to values of about $2 \mathrm{~nm}$.

The monochromated TEM also offers advantages for EFTEM spectrum imaging [9], because the microscope is equipped with a high resolution energy filter which is corrected for higher order spectral aberrations. In this work we show that EFTEM spectrum images can be recorded with an energy resolution less than $1 \mathrm{eV}$ for suitable small slit widths. However, at increased energy resolution aberrations, energy and spatial drift play a more pronounced role. Therefore we have developed new acquisition and correction algorithms that are necessary for measuring a reliable data set as well as for interpreting the data correctly and accurately (adaptive acquisition, spatial drift correction [10], simultaneous energy-drift, non-isochromaticity and spatial drift correction [11]. The spectral information now available with good energy resolution allows methods previously developed for EELS analysis now to be applied to map spectral features over a large field of view. Fig. 3 shows an EELS spectrum of GaN which was recorded with an EFTEM spectrum image in comparison with a spectrum acquired in spectroscopy mode. We will demonstrate the use of the methods with practical application examples from solid state technology.

\section{References}

[1] P.C. Tiemeijer, Inst. Phys .Conf. Ser. 161 (1999) 191.

[2] H.A. Brink et al., Microsc. Microanal. Vol. 7, Suppl. 2 (2001) 908.

[3] W. Grogger et al., Proc. 13 ${ }^{\text {th }}$ Eur. Microsc. Conf. (2003) Antwerp Vol.1, 271.

[4] K. Kimoto et al., Micron 36 (2005) 185.

[5] C. Mitterbauer et al., Ultramicroscopy 96 (2003) 469.

[6] G.A. Botton et al., Ultramicroscopy, in press. 
[7] S. Lazar et al. Ultramicroscopy 96 (2003) 535.

[8] F. Hofer et al., Proc. $13^{\text {th }}$ Eur. Microsc. Conf. (2003) Antwerp Vol.2, 725.

[9] G. Kothleitner, F. Hofer, Micron 34 (2003) 211.

[10] B. Schaffer et al., Ultramicroscopy 102 (2004) 27.

[11] B. Schaffer et al., Ultramicroscopy, in press.

[12] This work was financially supported by the special research program "Electroactive Materials", Austrian Science Fund (Vienna, Austria).

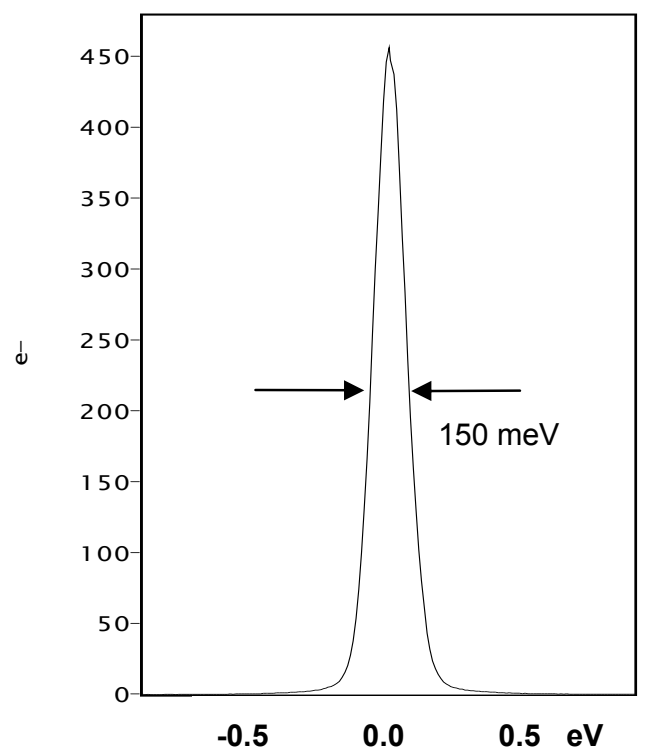

FIG.1 Zero-loss peak measured with the monochromated Tecnai F20 as installed in Graz, acquisition time $0.5 \mathrm{sec}$.

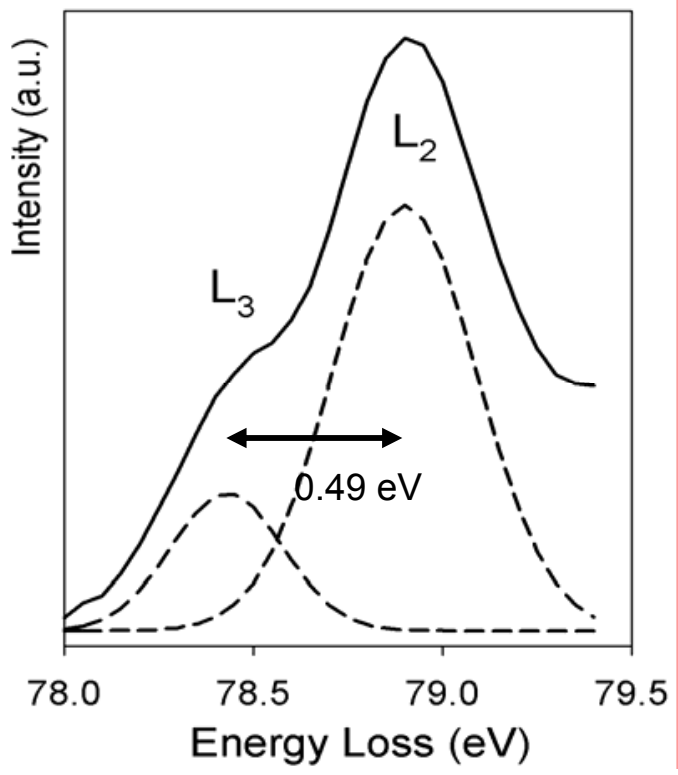

FIG.2 Al $\mathrm{L}_{2,3}$ ELNES of $\alpha-\mathrm{Al}_{2} \mathrm{O}_{3}$ fitted with Gaussians revealing the spin-orbit splitting (separation of $\mathrm{L}_{3}$ and $\mathrm{L}_{2}$ ).

FIG.3 Comparison of EELS spectra of $\mathrm{GaN}$ acquired in spectroscopy mode with a spectrum extracted from an EFTEM spectrum image of the same sample region.

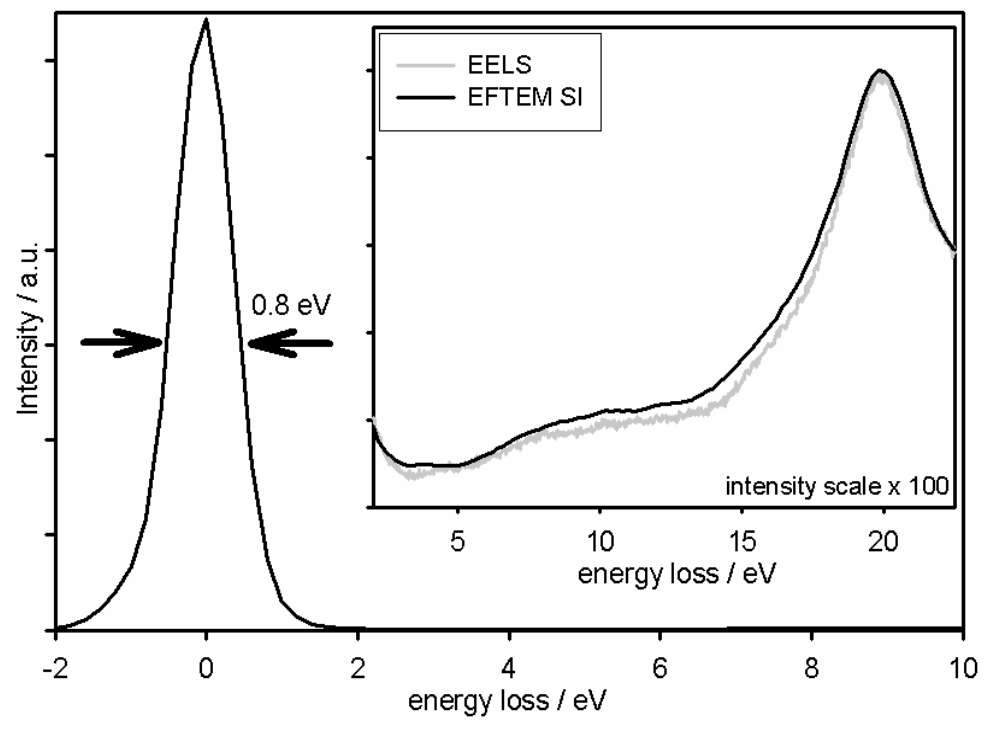

